\title{
Microsoft Natural Language Understanding System and Grammar Checker
}

\author{
Steve Richardson \\ Microsoft Research \\ One Microsoft Way \\ Redmond, WA 98052 \\ steveri@microsoft.com
}

The Natural Language Processing (NLP) group at Microsoft Research has been working for over five years on the development of a broad-coverage NL understanding system. In the last two years, the NLP group has been joined by the World Languages Research group, whose current mission is to extend the NLP group's technology to both European and Far-Eastern languages, and by the Natural Language Development group, which is responsible for transferring the technology into Microsoft products.

The presentation will provide a brief overview and demonstration of the components that comprise the Microsoft NLP technology, including the syntactic parser, logical form component, and lexical knowledge base (MindNet). There will also be a description of how the three NL groups at Microsoft have collaborated successfully to move the technology into real world products.

In particular, the grammar checker integrated in Microsoft Word 97 (also included in Office 97), which was released in January of this year, incorporates the NLP group's syntactic parser. It is envisioned that this very general and flexible component, together with the other components under development, will provide the basis for many NL-enabled functions as well as specific applications other than grammar checking.

The grammar checker will be demonstrated in the context of Word 97, showing a number of the errors it detects and some of the rewrites it suggests. Some usability and integration issues, such as background processing, will also be discussed. Finally, there will be a brief demonstration of the grammar checking functionality in the context of the more general NL understanding system.

Publications describing the work of the NLP group at Microsoft Research may be accessed on the World Wide Web at: http://www.research.microsoft.com/ 